\title{
Förderung von Managed Care und/oder «Listenmodellen»?
}

\author{
Stefan Kaufmann \\ Olivier Kappeler ${ }^{b}$ \\ a stv. Direktor, santésuisse \\ b Mitglied des Zentralvorstandes \\ der FMH
}

Die Förderung von Managed Care ist ein zentrales Thema der laufenden KVG-Revision. Interessierte Versicherer wie auch die Ärzteschaft wollen die in den letzten 10 Jahren eingeleitete Entwicklung aktiv vorantreiben. Die jeweiligen Grundhaltungen finden sich im Thesenpapier [1] der Ärztekammer, des obersten Organs der FMH, bzw. im Positionspapier [2] von santésuisse, dem Dachverband der Krankenversicherer.

In den letzten Monaten fand eine zunehmend polarisierende öffentliche Diskussion rund um die Listenmodelle verschiedener Versicherer (vgl. Kasten) statt. Dies ist insofern problematisch, als diese Modelle im gleichen Atemzug mit Managed-Care-Modellen genannt wurden.

In der Überzeugung, dass die offenen Fragen rund um die Entwicklung von Managed Care nur im Dialog gelöst werden können, hat die FMH zu einer Aussprache zwischen den verschiedenen Exponenten der Ärzteschaft und der Versicherer eingeladen. Das Gespräch fand am 28. März 2007 statt. Die Versicherer wurden dabei wie folgt vertreten: CSS (Reto Dahinden), Helsana (Philippe Signer, Petra Geiser, Georg Raguth), santésuisse (Stefan Kaufmann), SWICA (HansUeli Regius). Für die Ärzteschaft nahmen teil: FMH (Jacques de Haller, Olivier Kappeler), med-swiss.net (Max Albrecht Fischer), Ordine dei Medici (Franco Eugenio Denti, Francesca Gemnetti), Société Neuchâteloise de Médecine
(Pierre-Yves Bilat, Simone Walder-de Montmollin), Verein für Hausarztmedizin im Kanton Schaffhausen (Gerhard Schilling), Walliser Ärzteverband (Marc-Henri Gauchat). Der Anlass wurde von Peter Berchtold, Präsident Forum Managed Care, moderiert.

Ausgehend von den jeweiligen Positionen, diskutierten die Teilnehmer die Voraussetzungen, die nötig sind, um die Attraktivität und Wirksamkeit von Managed-Care-Modellen zu erhöhen, und wie solche Modelle durch Ärzteschaft und Versicherer einvernehmlich weiterentwickelt werden können.

Die Teilnehmer stimmen darin überein, dass die offenen Fragen im Dialog geklärt werden sollen. In den kommenden Monaten soll die Praktikabilität dieses Lösungsansatzes ausgelotet werden. Für Juni 2007 wird ein weiteres Gespräch vereinbart, bei dem eine Zwischenbilanz gezogen und weitere Schritte diskutiert werden.

Die Teilnehmer verabschiedeten sich in der Überzeugung, einen Lösungsansatz gefunden zu haben, der zwar noch fragil, aber doch mit dem nötigen Respekt belastbar sei.

\footnotetext{
Literatur

1 www.fmh.ch/shared/data/pdf/managed_care_d.pdf.

2 www.santesuisse.ch (Rubrik «Politik und Recht» $\rightarrow$ Positionspapiere).
}

\begin{abstract}
Listenmodelle werden kontrovers beurteilt. Während sich die betroffenen Versicherer auf den Standpunkt stellen, die Grundlage für diese Modelle sei im KVG gegeben (siehe unten), wehren sich die Ärztinnen und Ärzte der betroffenen Regionen und Kantone gegen die unilaterale Auswahl und bestreiten die Rechtmässigkeit dieser Modelle.
\end{abstract}

\section{Aktuell gültige Formulierung im KVG (Art. 41, Absatz 4)}

Die Versicherten können ihr Wahlrecht im Einvernehmen mit dem Versicherer auf Leistungserbringer beschränken, die der Versicherer im Hinblick auf eine kostengünstigere Versorgung auswählt (Art. 62, Abs. 1 und 3). Der Versicherer muss dann nur die Kosten für Leistungen übernehmen, die von diesen Leistungserbringern ausgeführt oder veranlasst werden; Ab- satz 2 gilt sinngemäss. Die gesetzlichen Pflichtleistungen sind in jedem Fall versichert.

Aktueller Stand der Diskussion dieses Artikels in der KVG-Revision (Position des Ständerates)

Auszug aus dem Amtlichen Bulletin SR Plenum Dezember 2006

Artikel 41a Antrag der Mehrheit

Titel: Managed Care

Abs. 1 Die Versicherten können ihr Wahlrecht auf Leistungserbringer beschränken, mit denen ihr Versicherer einen Vertrag über die Behandlung und deren Steuerung abgeschlossen hat (Managed Care). Die gesetzlichen Pflichtleistungen sind in jedem Fall versichert. 